\title{
A NEW METHOD OF IMPULSE NOISE REDUCTION IN GRAY AND COLOR IMAGES BY FUZZY FILTER
}

\author{
M.Harikrishnan \\ Department of Mathematics \\ Kongu Engineering College \\ Erode, India
}

\author{
R.Viswanathan \\ Department of Mathematics \\ Kongu Engineering College \\ Erode, India
}

\begin{abstract}
Image Reduction is one of the most important image processing tasks. In this article, a new fuzzy filter is presented for the removal of impulse noise in gray and color images. The filter consists of two stages. In the first stage, using trapezoid membership function four values are used to detect whether a given pixel contains noise or not. In the second stage fuzzy enhanced classifier is applied successively to remove the impulse noise in gray and color images. Experimental results are obtained to show the feasibility of the proposed approach. These results are also compared to other filters by numerical measures and visual inspection.
\end{abstract}

Keywords :Threshold, impulse noise, denoising, fuzzy filter, fuzzification, defuzzification

\section{INTRODUCTION}

Image processing has become a very important subject in electronic and computer engineering. Image processing has got wide varieties of applications in computer vision, multimedia communication, and television broadcasting etc., that demand very good quality of images.

\subsection{NOISE}

An image gets corrupted with noise during the process of acquisition, transmission, storage and retrieval. Noise may be classified as substitutive noise, impulsive noise (salt and pepper noise, random valued impulse noise etc.,) and additive noise e.g. .additive with Gaussian noise. Image restoration is one of the many areas it encompasses.

\subsubsection{Impulse Noise}

The probability density function of impulse noise is given by

$$
P(z)=\left\{\begin{array}{l}
P_{a} \text { for } z=a \\
P_{b} \text { for } z=b \\
0 \text { otherwise }
\end{array}\right.
$$

If $b>a$, gray level $b$ will appear as a light dot in the image. Impulse noise is generally digitized as pure white or black values in an image. If they are equal to the minimum and maximum allowed values in the digitized image, they are called standard values. As a consequence, negative impulses appear as black (pepper) points in an image. Positive impulse appears as white (salt) noise. In a 8 bit image, $a=0$ (black) and $\mathrm{b}=255$ (white).Fat fail distributed or impulse noise is sometimes called salt\& pepper noise or spike noise. An image containing salt $\&$ pepper noise will have dark pixels in bright regions \& bright pixels in dark regions. This type of noise can be caused by analog to digital convertor errors, bit errors in transmission etc.

1.2 IMAGE DENOISING

It is one of the main areas of image restoration. Image denoising is usually required to be performed before display or further processing like segmentation feature extraction, object recognition, texture analysis etc. The purpose of denoising is to suppress the noise quite efficiently while retaining the edges and other detailed features as much as possible. It becomes necessary to suppress the noise quite effectively without distorting the edges and the fine details of the image so that the filtered image becomes more useful for display and/or further processing. The nature of denoising depends on the type of the noise corrupting the image. S. Schulte et.al [1] proposed a filter removing random impulse noise using fuzzy random method and Stefan Schulte et.al [2] presented a fuzzy filter to remove noises in color images.. Morillas,S et al [3] presented fuzzy bilateral filtering for color images.In the literature [4]-[9], there are many methods available to remove impulse noise in gray scale and color images. Many filtering techniques over decades serve better for the enhancement of the images.Of these, median Filter is the non- linear filter in which sorting operation is performed on the input vector. The job is done as the mid value is taken as the input. Moving average filter overcomes the drawback of the median filter by using moving average in which the value at the central pixel is replaced by the mean value of the corresponding input neighborhood. Entropy filter denoises image by replacing every value by the information entropy of the values in its range $r$ neighborhood. The range filter allows to select a range of values. The left and right drag box can be used to change the lower and upper limit of the range, meaning that only rows with values within the chosen range remain in the visualization. An important feature of the range filter is that the values are distributed on a linear scale according to the values of the data. Standard filter filters image by replacing every value by the standard deviations of the values in its range $r$ neighborhood. This paper deals with a new fuzzy filtering technique for the removal of impulsive noise in both gray and color images. Fuzzy filters are easy to realize by means of simple fuzzy rules.

\section{PROPOSED NEW FUZZY FILTER FOR IMPULSE NOISE REDUCTION IN GRAY AND COLOR IMAGES}

This paper focuses on developing a new filter to remove impulse noise in gray and color images. The proposed filter uses fuzzy trapezoid membership function to remove impulse noise in the image. Let $\mathrm{f}(\mathrm{x}, \mathrm{y})$ and $\mathrm{f}^{\prime}(\mathrm{x}, \mathrm{y})$ denote the pixel value at location $(\mathrm{x}, \mathrm{y})$ 
of the given input image and the image with the noise respectively.

The impulse noise detection with noise $\alpha$ is given as follows

$$
f(x, y)=\left\{\begin{array}{c}
£_{x, y} \text { with theprobabiliy } \alpha \\
Q_{x, y} \text { with probability of } 1-\alpha
\end{array}\right.
$$

where $\mathrm{f}_{\mathrm{X}_{\mathrm{v}} \mathrm{y}}$ denotes the noise value

$$
\operatorname{Deten}_{\mathrm{i}}=\operatorname{abs}\left(\mathrm{f}_{\mathrm{x}}{ }^{\prime}-\mathrm{f}_{\mathrm{y}}\right)
$$

By using fuzzy trapezoid membership classification, four values are used to detect whether a given pixel contains noise or not. The trapezoid membership classification is used because the deployment of the trapezoidal membership function is better than the triangular membership function. In addition, trapezoidal function provides the optimal degree of compatibility for higher levels of attributes than the triangular function. At the same time, it is provided with the maximum membership value which corresponds to the centroid of the triangular shape. As a result, the application of trapezoidal membership function using enhanced fuzzy classifier gives higher degrees of compatibility resulting in the approximation of the desired output. To use trapezoidal membership function, four parameters are necessary as given in figure2.1, and so 4 x 4 matrix is used.

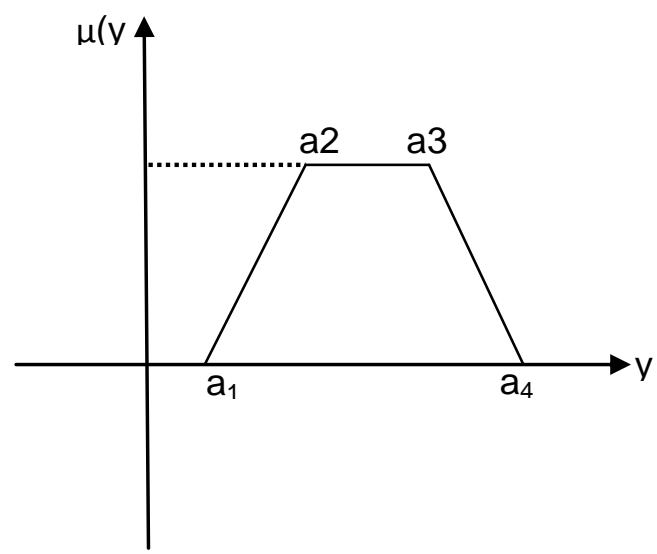

Figure 2.1 Trapezoidal membership function

By applying the trapezoidal membership function, initially, Deten $\mathrm{n}_{\mathrm{i}}$ is mapped onto range $[0,100]$ and are classified into two partitions. The two partitions comprise low $\operatorname{Deten}_{\mathrm{i}}(\mathrm{L})$ and high intensity $\operatorname{Deten}_{\mathrm{i}}(\mathrm{H})$ respectively using two different thresholds, namely $\mathrm{T}_{1}$ and $\mathrm{T}_{2}$.

If $\left(\right.$ Deten $_{\mathrm{i}}$ is in range $\left(0, \mathrm{~T}_{2}\right)$

\{pixel is classified as $\left.\operatorname{Deten}_{\mathrm{i}}(\mathrm{L})\right\}$ )

Else

If $\left(\right.$ Deten $_{i}$ is in range $\left(\mathrm{T}_{1}, 100\right)$

$\left\{\right.$ pixel is classified as $\left.\operatorname{Deten}_{\mathrm{i}}(\mathrm{H})\right\}$ )

Endif

Let us consider a scenario, a process followed to restore the noisy images.

Table 2.1 Impulse noise reduction process

\begin{tabular}{|l|l|l|l|}
\hline 240 & 120 & 120 & 120 \\
\hline 240 & 120 & 120 & 120 \\
\hline 240 & 120 & 120 & 120 \\
\hline 240 & 120 & 120 & 120 \\
\hline
\end{tabular}

\begin{tabular}{|l|l|l|l|}
\hline 240 & 250 & 180 & 120 \\
\hline 240 & 120 & 120 & 120 \\
\hline 240 & 120 & 120 & 120 \\
\hline 240 & 120 & 120 & 120 \\
\hline
\end{tabular}

\begin{tabular}{|l|l|l|l|}
\hline 240 & 120 & 160 & 120 \\
\hline 240 & 120 & 120 & 120 \\
\hline 240 & 120 & 120 & 120 \\
\hline 240 & 120 & 120 & 120 \\
\hline
\end{tabular}

(c) noisy image removed

\begin{tabular}{|l|l|l|l|}
\hline 240 & 120 & 120 & 120 \\
\hline 240 & 120 & 120 & 120 \\
\hline 240 & 120 & 120 & 120 \\
\hline 240 & 120 & 120 & 120 \\
\hline
\end{tabular}

(d) noisy image completely removed

The motivation for Impulse noise detection and filtering using Fuzzy Classification of noisy images is illustrated as follows. In Table2.1(a), the original image of a pixel is shown. Let us consider that the two pixels in the image block comprise of impulse noise with the respective noisy pixel values as 250 and 180 as illustrated in Table 2.1(b). Then, in this case scenario, let us assume that the locations of these two impulse noisy pixels are detected and replaced using enhanced fuzzy classification as shown in Table 2.1(c).

When compared with Table 2.1(a) and Table2.1(c), one noisy pixel is restored after application of first membership function. The impulse noisy pixel value 180 is replaced with the value of 160 which also comprise of impulse noise pixel value when compared to the actual original pixel value of the corresponding image. Subsequently, if we repeat the same process by applying the membership function, then both noisy pixels are removed as illustrated in Table2.1 (d).

The original cameraman image, the impulse noise image is shown in figure 2.2 .

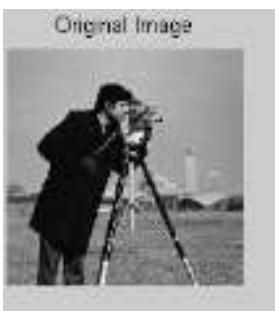

(a) Original Cameraman image

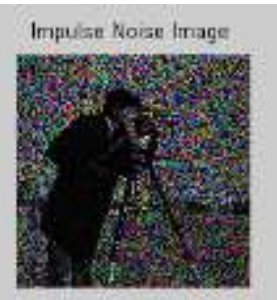

(b) Impulse noise image

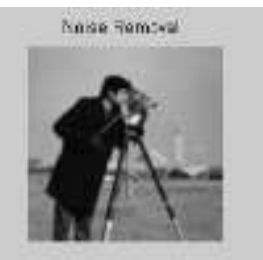

(c) Noise Removal using Enhanced Fuzzy Classifier

Figure 2.2 Impulse Noise Reduction Process

The quality of images using fuzzy classification is measured by means of peak signal-to-noise ratio (PSNR), detection accuracy and noise level.

\section{Experimental Results Impulse noise detection} and filtering using Enhanced Fuzzy Classifier

In order to assess the performance of the proposed Impulse Noise Removal using Enhanced Fuzzy Classifier(INREFC), extensive simulations are carried out on standard gray and color images of cameraman under different noisy conditions in MATLAB. The performance is measured in terms of Peak Signal-to-Noise Ratio (PSNR) detection accuracy and noise level.

PSNR is defined as

$$
\text { PSNR }=10 \log _{10}\left(\frac{255^{2}}{\mathrm{MSE}}\right) \mathrm{dB}
$$


Detection accuracy is defined as

$$
\text { Detection Accuracy }=\sqrt{\frac{1}{\mathrm{~N}} \Sigma\left(\mathrm{x}_{\mathrm{i}}^{2}+\mathrm{y}_{\mathrm{i}}^{2}\right)} \mathrm{i}=1,2, \ldots \mathrm{N}
$$

\subsection{Measure of PSNR}

The table 1 given below shows the peak signal-to-noise rate for different image sizes which is obtained using gray images of cameraman.

Table 3.1 Measure of PSNR

\begin{tabular}{|c|c|c|c|c|}
\hline \multirow{2}{*}{$\begin{array}{c}\text { Image Size } \\
\text { (pixels) }\end{array}$} & \multicolumn{4}{|c|}{ PSNR (dB) } \\
\cline { 2 - 5 } & $\begin{array}{c}\text { Proposed } \\
\text { INREFC }\end{array}$ & $\begin{array}{c}\text { Entropy } \\
\text { Filter }\end{array}$ & $\begin{array}{c}\text { Range } \\
\text { Filter }\end{array}$ & $\begin{array}{c}\text { Standard } \\
\text { Filter }\end{array}$ \\
\hline 150 & 93 & 90.25 & 89.5 & 86.25 \\
\hline 200 & 93.55 & 92.55 & 90.25 & 85 \\
\hline 250 & 95 & 94.5 & 93.35 & 88 \\
\hline 300 & 96.5 & 96 & 94.55 & 90 \\
\hline
\end{tabular}

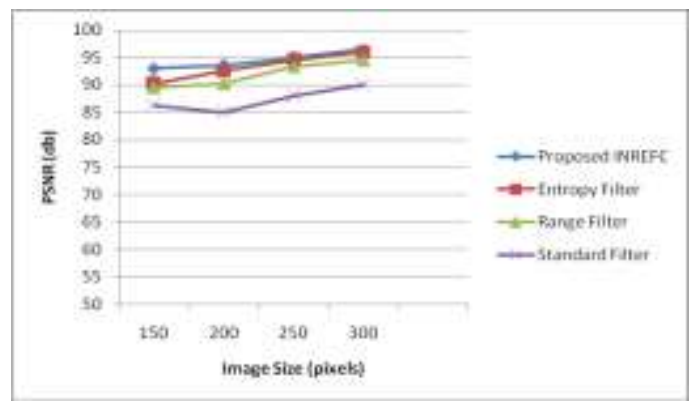

Figure 3.1 Image Size Vs PSNR

The figure 3 shows the measure of PSNR for both color and gray images for impulse noise detection and filter using enhanced fuzzy classifier. A detailed comparison analysis is made using three other filters, standard filter, range filter and entropy filter. From the figure, it is evident that the peak signal-to-noise ratio is comparatively higher using enhanced fuzzy classifier when compared to other three filters. The PSNR rate is high using enhanced fuzzy classifier due to the fact that trapezoid membership classification is used with a variance of $3-5 \%$ of signal-to-noise ratio increased when compared to entropy filter, $5 \%$ of signal-to-noise ratio increased when compared to range filter.

\subsection{Measure of Detection Accuracy}

The table 3.2 given below shows the detection accuracy rate for different image sizes which is obtained using color and gray images of cameraman.

Table 3.2 Measure of Detection Accuracy

\begin{tabular}{|c|c|c|c|c|}
\hline \multirow{2}{*}{$\begin{array}{c}\text { Image } \\
\text { Size } \\
\text { (pixels) }\end{array}$} & $\begin{array}{c}\text { Proposed } \\
\text { INREFC }\end{array}$ & $\begin{array}{c}\text { Entropy } \\
\text { Filter }\end{array}$ & $\begin{array}{c}\text { Range } \\
\text { Filter }\end{array}$ & $\begin{array}{c}\text { Standard } \\
\text { Filter }\end{array}$ \\
\hline 150 & 65 & 58 & 55 & 53 \\
\hline 200 & 70 & 60 & 58 & 55 \\
\hline 250 & 72 & 65 & 60 & 52 \\
\hline 300 & 75 & 68 & 62 & 54 \\
\hline
\end{tabular}

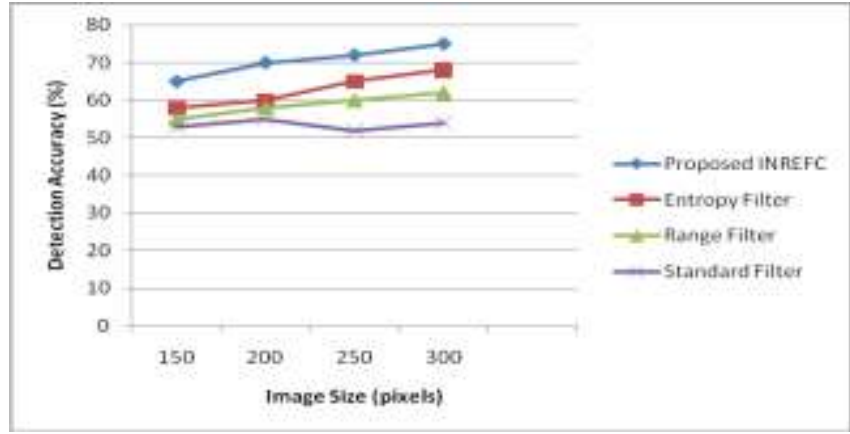

Figure 3.2 Image Size Vs Accuracy

Figure 3.2 describes the accuracy level of both color and gray image of cameraman using Enhanced Fuzzy Classifier. The detection accuracy level of the image is measured in terms of \%. When the size of image which is measured in terms of pixels gets increased, the detection accuracy is reduced. But when compared with three other methods, enhanced fuzzy classifier achieves higher detection accuracy since it maintained two partitions, low detention ( $\mathrm{L}$ ) and high detention $(\mathrm{H})$ to obtain the detection accuracy level. The variance in the detection accuracy level is 8$10 \%$ high in the proposed Impulse noise detection and filtering using Enhanced Fuzzy Classifier.

\subsection{Measure of Noise Level}

The table 3.3.1 given below shows the level of noise present for different image sizes which is obtained using gray image of cameraman.

Table 3.3.1 Measure of Noise level

\begin{tabular}{|c|c|c|c|c|}
\hline \multirow{2}{*}{$\begin{array}{c}\text { Image Size } \\
\text { (pixels) }\end{array}$} & $\begin{array}{c}\text { Proposed } \\
\text { INREFC }\end{array}$ & $\begin{array}{c}\text { Entropy } \\
\text { Filter }\end{array}$ & $\begin{array}{c}\text { Range } \\
\text { Filter }\end{array}$ & $\begin{array}{c}\text { Standard } \\
\text { Filter }\end{array}$ \\
\hline 150 & 75 & 78 & 80 & 85 \\
\hline 200 & 72 & 78 & 80 & 80 \\
\hline 250 & 60 & 70 & 71 & 75 \\
\hline 300 & 59 & 64 & 69 & 70 \\
\hline
\end{tabular}

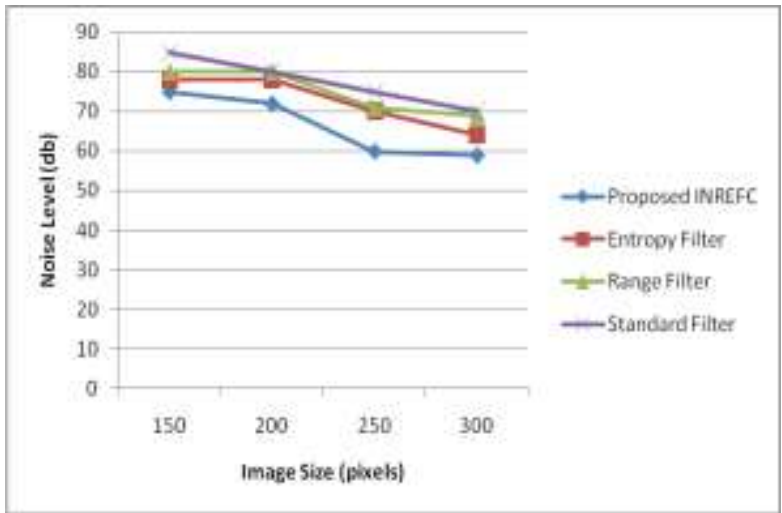

Figure 3.3.1. Image Size Vs Noise Level

Figure 3.3.1 describes the noise level of color and gray image applied to image of cameraman using Enhanced Fuzzy Classifier. The level of noise present in the color and gray images is measured in terms of decibels. The level of noise occurred in proposed INREFC is lesser when compared to other three methods due to the fact that the noise is removed using fuzzification and defuzzification process. The variance in the noise level is reduced $4-8 \%$ in the proposed Impulse noise detection and filtering using Enhanced Fuzzy Classifier when compared to entropy filter, 
$5-10 \%$ reduced when compared to range filter.

\section{CONCLUSION}

An enhanced approach has been proposed for the removal of impulse noise using enhanced fuzzy classifier. The main advantages of this filter are the denoising capability and suitability for both gray and color images. A numerical measure ,such as PSNR, detection accuracy, noise level have shown convincing results. Moreover, we have shown that the proposed method achieves a more comparable noise reduction performance than the other existing filters. Our future work will be on focusing reduction of speckle noise, stripping noise etc., in gray and color images.

\section{REFERENCES}

[1] Schulte,S V.De Witte, Nachtegael,M D.Van der Weken, and Kerre EE .2006. "Fuzzy random impulse noise reduction method", Fuzzy Sets and Systems vol 158 no 3 pp 270-283

[2] Schulte,S, Morillas,VGregori, Vand Kerre,EE .2007. "A new fuzzy color correlated impulse noise reduction Method" IEEE. Transactions on image Processing, Vol 16 No10, pp $2565-2575$.

[3] Morillas,S Gregori V and Sapena,A.2006."Fuzzy bilateral filtering for color images",Lecture Notes Comput.Sci.,vol 4141,pp 138-145.

[4] Schulte, S V. De Wite, Nachtegael M, Van den weken,D and Kerre EE.2006."Fuzzy Two step filter for impulse noise reduction from color images", IEEE.Transactions on image processing,vol 15 .

[5] Russo, F. 1999 ."Fire operators for image processing,” Fuzzy Sets Syst., vol.103, no. 2, pp. 265-275.

[6] Dimitri Van De Ville, Mike Nachtegael, Van der Weken, Etienne E.Kerre, Wilfried Philips and,Ignace Lemahieu.2003."NoiseReduction by Fuzzy Image Filtering.", IEEE Transactions on Fuzzy Systems, Vol.11, No.4 .

[7] Nachtegael, $M$ and Kerre, EE, Fuzzy mathematical morphology: state of the art,in:E.E. Kerre M.Nachtegael (Eds.) .2000. "Fuzzy Techniques in Image Processing, Studies in Fuzziness and Soft computing", vol.52, Springer Verlag,Heidelberg,, pp 3-57.

[8] Lee, CS , Kuo VH \& Yu PT. 1997."Weighted fuzzy mean filters for image processing", Fuzzy sets and Systems, vol 89 , pp. $157-180$.

[9] S.Schulte, B.Huysmans,A.Pizurica,EE,Kerre and W.Philips .2006."A New Fuzzy Based wavelet shrinkage image denoising technique", Lecture Notes Computer science vol 41no.79 pp 12-23. 